\title{
On Refutation Rules
}

\author{
Tomasz Skura
}

\begin{abstract}
The goal of this paper is to generalize specific techniques connected with refutation rules involving certain normal forms. In particular, a method of axiomatizing both a logic $L$ and its complement $-L$ is introduced.
\end{abstract}

Mathematics Subject Classification (2000). Primary 03B22; Secondary 03B45.

Keywords. Refutation systems, axiomatization, refutation search, modal logic.

\section{Introduction}

Refutation rules are rules preserving non-validity. Typical examples are the following (introduced by Łukasiewicz $[2,3]$ ).

( $R_{s}$ or reverse substitution) $s(A) / A$ where $s(A)$ is a substitution instance of $A$ ( $R_{m p}^{L}$ or reverse modus ponens) $B / A$ where $A \rightarrow B \in L$

( $R_{d}$ or the disjunction property) $A \quad B / A \vee B$

Note that $R_{s}, R_{m p}^{L}$ are refutation rules for every logic closed under substitution and modus ponens, while $R_{d}$ is a refutation rule for Intuitionistic Logic $(I N T)$ but it is not a refutation rule for Classical Logic $(C L)$.

A pair consisting of a set of refutation axioms (which are some non-valid formulas) and a set of refutation rules is a refutation system. It is an axiom system, just like traditional axiom systems, but it generates non-valid formulas rather than valid ones. Of course, axiom systems are notoriously bad for proof search. And so are refutation systems containing the rules: $R_{s}$ and $R_{m p}^{L}$ (although they have some interesting theoretical aspects (see $[5,6])$ ). However, the rule $R_{d}$ is more promising for proof search (or, rather, refutation search). It has the important property that each premise is simpler than the conclusion. Also, in INT for example, we have the following.

$A \vee B \notin I N T$ iff both $A \notin I N T$ and $B \notin I N T$.

Thus, you can reduce $A \vee B$ to $A$ and $B$, when you search for a refutation of $A \vee B$. 
In this paper we study refutation rules of this kind. We are interested in refutation rules of the form

$$
\frac{F_{1} \cdots F_{k}}{F}
$$

where $F$ is a certain normal form of rank $n>0$ and each $F_{i}$ is a normal form of rank $<n$. Specific rules of this kind can be extracted from $[4,6]$. Here we generalize these techniques obtaining a simple method of proving that a refutation system is complete for a logic $L$ (that is, it axiomatizes the complement $-L$ of $L$ ). This also gives an axiomatization for $L$ by some rules dual to the refutation rules. What is more, our method provides a simple refutation-search procedure that is both label-free and cycle-free. Thus, it may be simpler than standard refutation methods, in which refuting a formula is justified by failure to find a proof for it. Our concepts will be illustrated in the modal logic $S 4$, where the problem of refutation search is non-trivial.

\section{Refutation Rules}

Let $L$ be a propositional logic (which can be thought of as the set of propositional formulas valid in structures of some sort).

Our refutation rules involve $L$ normal forms having the following propery. For every formula $A$, there are $L$ normal forms $A^{1},, A^{l}$ such that $A \in L$ iff $A^{1} \in L, \ldots, A^{l} \in L$.

(Note that $A^{i} / A$ is a refutation rule for $L$, and $A^{1}, \ldots, A^{l} / A$ is a proof rule for $L$.)

For example, classical conjunctive normal forms are of this kind. However, normal forms based on clausal forms (see [4,6]) are more useful. They are both simple and general. We assume that a natural number $r(F)$ (called the rank of $F$ ) has been assigned to every normal form $F$, and some (simple) normal forms are of rank 0.

(Here for any finite sets $X, Y$ of formulas, the symbol $X \longrightarrow Y$ stands for $\bigwedge X \rightarrow \bigvee Y$, where $\bigwedge X(\bigvee X)$ is a conjunction (a disjunction) of the formulas in $X$, and $\square X=\{\square A: A \in X\}$.)

Example 2.1. (For more details, see [4,6].) An $S 4$ normal form $F$ is either a $\square$-free formula or a formula

$$
\square S \longrightarrow a_{1}
$$

where $S=\Theta \cup \Delta \cup \Gamma$

$$
\begin{aligned}
& \Theta=\left\{\square a_{i} \equiv c_{0}: 1 \leq i \leq m\right\} \\
& \Delta=\left\{\square b_{j} \equiv c_{j}: 1 \leq j \leq n\right\}
\end{aligned}
$$

$\Gamma$ is a finite set of $\square$-free formulas, all $a_{i}, b_{j}, c_{0}, c_{j}$ are propositional variables, and $m>0, n \geq 0$.

The rank $r(F)$ of $F$ is 0 if $F$ is $\square$-free, and

$r(F)=n+1$ if $F=\square S \longrightarrow a_{1}$. (Note that the number of formulas in $\Theta$ is ignored.) 
For every formula $A$, its corresponding $S 4$ normal form $A^{\prime}$ can be constructed with the property that $A \in S 4$ iff $A^{\prime} \in S 4$.

Definition 2.2. A refutation rule for a $\operatorname{logic} L$ is a rule of the kind

$$
X / F
$$

where $F$ is an $L$ normal form of rank $>0$ and $X$ is a finite non-empty set of $L$ normal forms of rank $<r(F)$ having the following property.

If $X \subseteq-L$ then $F \in-L$.

(This is usually established by some semantic (or algebraic) argument.)

Example 2.3. The following is a refutation rule for $S 4$ [see [6]].

$$
\left(R_{S 4}\right) \quad \frac{C_{1} \ldots C_{m} F_{1} \ldots F_{n}}{F}
$$

where $F$ is an $S 4$ normal form of rank $>0$, and

$$
\begin{aligned}
& C_{i}=\Gamma \longrightarrow a_{i}, c_{0}, c_{1}, \ldots, c_{n} \quad(1 \leq i \leq m) \\
& F_{j}=\square\left(\square b_{j} \equiv c_{j}\right), \square \Delta_{j}^{-}, \square \Gamma, \square a_{1}, \ldots, \square a_{m}, \square c_{0} \longrightarrow b_{j} \\
& \Delta_{j}^{-}=\Delta-\left\{\square b_{j} \equiv c_{j}\right\} \quad(1 \leq j \leq n)
\end{aligned}
$$

Note that all $C_{i}$ are of rank 0 , and each $F_{j}$ is an $S 4$ normal form of rank $<r(F)$. Moreover $F_{j}$ is $S 4$-equivalent to

$$
F_{j}^{\prime}=\square S, \square a_{1} \longrightarrow b_{j} .
$$

(We say that formulas $A, B$ are $S 4$-equivalent iff $A \equiv B \in S 4$.)

Definition 2.4. (i) A refutation system $\mathbf{R}$ for a logic $L$ is a pair $(R A, R R)$, where $R R$ is a set of refutation rules for $L$ and $R A$ is the set of refutation axioms for $L$ (that is, the set of all normal forms $F$ of rank 0 such that $F \notin L)$.

(ii) A normal form is $\mathbf{R}$-refutable iff it is derivable from $R A$ by $R R$.

Note that if $F$ is $\mathbf{R}$-refutable, then $F \notin L$. Thus, a refutation system generates non-valid normal forms by derivations. Of course, it need not be complete (that is, having the property that every $F \notin L$ is $\mathbf{R}$-refutable).

\section{Completeness}

When is a refutation system for $L$ complete? We introduce a condition (called the reduction property) that proves sufficient.

Definition 3.1. Let $\mathbf{R}$ be a refutation system for a logic $L$. $\mathbf{R}$ has the reduction property iff for every normal form $F$ of rank $>0$, the following conditions are satisfied.

(i) The set $R R(F)$ of refutation rules of the form $X / F$ (that is, the set $\{X / F: X / F \in R R\})$ is non-empty and finite.

(ii) If $F \in-L$ then there is a rule $X / F \in R R(F)$ such that $X \subseteq-L$.

The reduction property also provides a proof system axiomatizing $L$. 
Definition 3.2. Let $\mathbf{R}$ be a refutation system for a logic $L$ with the reduction property.

(i) The set of proof rules for $L$ corresponding to $R R$ is the set $P R R=$ $\left\{A_{1}, \ldots, A_{t} / F: F\right.$ is a normal form of rank $>0$, each $A_{i} \in X_{i}$ and $\left.\left\{X_{1} / F, \ldots X_{t} / F\right\}=R R(F)\right\}$.

(Note that $L$ is closed under the rules of $P R R$.)

(ii) The set of proof axioms for $L$ corresponding to $R A$ is the set $P R A$ of all normal forms $F$ of rank 0 such that $F \in L$.

(iii) The proof system for $L$ corresponding to $\mathbf{R}$ is the pair $\mathbf{P R}=$ (PRA, PRR).

We simply say " $F$ is provable" (in symbols $\vdash F$ ) instead of " $F$ is derivable from $P R A$ by $P R R$ ". Observe that if $\vdash F$ then $F \in L$. And we say " $F$ is refutable" (in symbols $\dashv F$ ) instead of " $F$ is derivable from $R A$ by $R R$ ". Of course, if $\dashv F$ then $F \notin L$.

Theorem 3.3. Let $\mathbf{R}$ be a refutation system for a logic $L$ with the reduction property. Then for every $L$ normal form $F$ we have:

(i) Either $\vdash F$ or $\dashv F$.

(ii) If $F \notin L$ then $\dashv F$.

(iii) If $F \in L$ then $\vdash F$.

Proof. (i) By induction on $r(F)$.

(1) $r(F)=0$. Either $F \in L$ or $F \notin L$. Hence $\vdash F$ or $\dashv F$.

(2) $r(F)>0$ and this is true for $L$ normal forms of rank $<r(F)$.

Since $\mathbf{R}$ has the reduction property, $R R(F)$ is non-empty and finite. Let $X_{1} / F, \ldots, X_{t} / F$

be all rules in $R R(F)$. Consider any $A \in X_{i}(1 \leq i \leq t)$. $A$ is of rank $<r(F)$, so by the induction hypothesis, $A$ is provable or $A$ is refutable.

(Case 1) For some $1 \leq i \leq t$, every $A \in X_{i}$ is such that $\dashv A$. Then $\dashv F$ by $R R$.

(Case 2) For every $1 \leq i \leq t$, some $A \in X_{i}$ is such that $\vdash A$. Then $\vdash F$ by $P R R$.

Therefore either $\vdash F$ or $\dashv F$.

(ii) Assume that $F \notin L$. Then it is not the case that $\vdash F$. (Otherwise $F \in L$.) Hence $\dashv F$ by (i).

(iii) Assume that $F \in L$. Then it is not the case that $\dashv F$. (Otherwise $F \notin L$.) Hence $\vdash F$ by (i), as required.

Remark 3.4. In a specific logic, the proof system PR can be replaced by some elegant proof system by showing that both $P R A$ and $P R R$ are derivable in that system.

Example 3.5. Let $\mathbf{R}$ consist of the following (see [6]).

$R A$ : All $\square$-free formulas that are not in $C L$.

$R R: R_{S 4}$ and

$\begin{array}{ll}\left(R G_{j}\right) & \frac{G_{j}}{F} \\ \left(R H_{j}\right) & \frac{H_{j}}{F}\end{array}$ 
where $F$ is an $S 4$ normal form of rank $>0$ and

$$
\begin{aligned}
G_{j} & =\square \Theta, \square \Delta_{j}^{-}, \square \Gamma, \square b_{j}, \square c_{j} \longrightarrow a_{1} \\
H_{j} & =\square \Theta, \square\left(\square b_{j} \equiv c_{0}\right), \square \Delta_{j}^{-}, \square \Gamma, \square\left(c_{0} \equiv c_{j}\right) \longrightarrow a_{1} \quad(1 \leq j \leq n)
\end{aligned}
$$

Note that $G_{j}, H_{j}$ are $S 4$ normal forms of rank $<r(F)$, and they are $S 4$-equivalent to $G_{j}^{\prime}, H_{j}^{\prime}$, respectively, where

$$
\begin{aligned}
G_{j}^{\prime} & =\square S, \square b_{j} \longrightarrow a_{1} \\
H_{j}^{\prime} & =\square S, \square\left(c_{0} \equiv c_{j}\right) \longrightarrow a_{1}
\end{aligned}
$$

We remark that $\mathbf{R}$ is a refutation system for $S 4$ (see [6]), and it has the reduction property. Indeed, 3.1(i) is obvious. To show 3.1(ii), note the following fact.

If (some $C_{i} \in S 4$ or some $F_{j} \in S 4$ ) and all $G_{1}, \ldots, G_{n}, H_{1}, \ldots, H_{n} \in S 4$, then $F \in S 4$ (see [6]).

Hence if $F \notin S 4$, then (all $C_{j} \notin S 4$ and all $F_{j} \notin S 4$ ) or some $G_{j} \notin S 4$ or some $H_{j} \notin S 4$.

Therefore (by 3.3) $\mathbf{R}$ is complete for $S 4$.

\section{Refutation Search}

Let $\mathbf{R}$ be a refutation system for a logic $L$ with the reduction property. Then for any $L$ normal form $F$ of rank $>0$ we have:

$$
F \in-L \text { iff } X_{1} \subseteq-L \text { or } \ldots \text { or } X_{t} \subseteq-L
$$

where $\left\{X_{1} / F, \ldots, X_{t} / F\right\}=R R(F)$.

This fact provides the following simple refutation-search procedure for normal forms of rank $>0$.

Write $F$.

Then write $X_{1}, \ldots, X_{t}$.

Every formula in each $X_{i}$ is a normal form of rank $<r(F)$, so by repeating such reductions, the procedure terminates at some normal forms of rank 0 , which are either refutation axioms or proof axioms. From them either a refutation for $F$ or a proof for $F$ can be obtained (see the proof of 3.3(i)). (Of course, for any formula $A$, its corresponding normal forms $A^{1}, \ldots, A^{l}$ can be constructed, so that this is also a refutation-search procedure for any $A$.)

Example 4.1. Let $F=\square S \longrightarrow p_{1}$, where

$$
\begin{aligned}
& S=\Theta \cup \Gamma \\
& \Theta=\left\{\square p_{1} \equiv q, \square p_{2} \equiv q, \square p_{3} \equiv q\right\} \\
& \Gamma=\left\{p_{1} \vee p_{2}, p_{1} \vee p_{3}, p_{2} \vee p_{3}\right\}
\end{aligned}
$$

and $p_{1}, p_{2}, p_{3}, q$ are propositional variables.

\section{Refutation}

We have $n=0$, so $F$ is obtained by $R_{S 4}$ from

$$
\begin{aligned}
& C_{1}=\Gamma \longrightarrow p_{1}, q \\
& C_{2}=\Gamma \longrightarrow p_{2}, q
\end{aligned}
$$




$$
C_{3}=\Gamma \longrightarrow p_{3}, q
$$

because each $C_{i} \notin C L$.

\section{Conclusions}

Our method is complementary to standard proof-search procedures. It is a direct refutation method, that is, refuting $A$ is justified by the existence of a derivation, whereas standard decision methods provide indirect refutation procedures, in which refuting $A$ is justified by failure to find a proof for $A$.

Our method is based on reductions and it is cycle-free, so it may be useful in logics for which tableau procedures are based on repetitions and cycles, for example in transitive modal logics (for more information, see [1]).

It may also be useful in logics for which simple contraction-free sequent systems are not easy to find (for a discussion, see [6]).

Our method is interesting because of practical applications as well as theoretical ones.

Open Access. This article is distributed under the terms of the Creative Commons Attribution Noncommercial License which permits any noncommercial use, distribution, and reproduction in any medium, provided the original author(s) and source are credited.

\section{References}

[1] Goré, R.: Tableau methods for modal and temporal logics. In: Agostino, M.D., Gabbay, D., Hähnle, R., Posega, J. (eds.) Handbook of Tableau Methods, pp. 297-396. Kluwer, Dordrecht (1999)

[2] Łukasiewicz, J.: Aristotle's Syllogistic from the Standpoint of Modern Formal Logic. Clarendon Press, Oxford (1951)

[3] Łukasiewicz, J.: On the intuitionistic theory of deduction. Indagationes Mathematicae 14, 202-212 (1952)

[4] Skura, T.: Refutations, proofs, and models in the modal logic K4. Studia Logica 70, 193-204 (2002)

[5] Skura, T.: A refutation theory. Logica Universalis 3, 293-302 (2009)

[6] Skura, T.: Refutation systems in propositional logic. In: Gabbay, D., Guenthner, F. (eds.) Handbook of Philosophical Logic, vol. 16, pp. 115-157 (2011)

Tomasz Skura

Institute of Philosophy

University of Zielona Góra

Wojska Polskiego 71A

65-762 Zielona Góra

Poland

e-mail: T.Skura@ifil.uz.zgora.pl;

tskura@uz.zgora.pl

Received: August 30, 2010.

Accepted: August 15, 2011. 